\title{
Suppression of Acoustic Streaming in Shape-Optimized Channels
}

\author{
Bach, Jacob S.; Bruus, Henrik
}

Published in:

Physical Review Letters

Link to article, DOI:

10.1103/PhysRevLett.124.214501

Publication date:

2020

Document Version

Publisher's PDF, also known as Version of record

Link back to DTU Orbit

Citation (APA):

Bach, J. S., \& Bruus, H. (2020). Suppression of Acoustic Streaming in Shape-Optimized Channels. Physical Review Letters, 124(21), [214501]. https://doi.org/10.1103/PhysRevLett.124.214501

\section{General rights}

Copyright and moral rights for the publications made accessible in the public portal are retained by the authors and/or other copyright owners and it is a condition of accessing publications that users recognise and abide by the legal requirements associated with these rights.

- Users may download and print one copy of any publication from the public portal for the purpose of private study or research.

- You may not further distribute the material or use it for any profit-making activity or commercial gain

- You may freely distribute the URL identifying the publication in the public portal

If you believe that this document breaches copyright please contact us providing details, and we will remove access to the work immediately and investigate your claim. 


\title{
Suppression of Acoustic Streaming in Shape-Optimized Channels
}

\author{
Jacob S. Bach ${ }^{*}$ and Henrik Bruus $\odot^{\dagger}$ \\ Department of Physics, Technical University of Denmark, DTU Physics Building 309, DK-2800 Kongens Lyngby, Denmark
}

(Received 18 January 2020; accepted 8 May 2020; published 27 May 2020)

\begin{abstract}
Acoustic streaming is an ubiquitous phenomenon resulting from time-averaged nonlinear dynamics in oscillating fluids. In this theoretical study, we show that acoustic streaming can be suppressed by two orders of magnitude in major regions of a fluid by optimizing the shape of its confining walls. Remarkably, the acoustic pressure is not suppressed in this shape-optimized cavity, and neither is the acoustic radiation force on suspended particles. This basic insight may lead to applications, such as acoustophoretic handling of $\mathrm{nm}$-sized particles, which is otherwise impaired by the streaming.
\end{abstract}

DOI: 10.1103/PhysRevLett.124.214501

When a fluid executes oscillatory motion due to acoustics or vibrating boundaries, the inherent fluid-dynamical nonlinearities spawn a steady flow adding to the oscillatory motion. This phenomenon, called acoustic streaming, has a rich, 200 year old history. Observations by Ørsted (1809) and Savart (1827) of different motions of coarse and fine grained powders over vibrating Chladni plates, were in 1831 conclusively attributed to acoustic streaming in the air by Faraday in his seminal experiments on Chladni plates placed in a partial vacuum [1]. In 1876, Dvořák observed acoustic streaming caused by standing sound waves in Kundt's tubes [2]. A theoretical explanation of this boundary-induced streaming in various geometries was provided in 1884 by Lord Rayleigh in terms of an oscillatory boundary-layer flow, which by time averaging induces a steady slip velocity near the boundary that drives the steady streaming [3]. In 1932, Schlichting characterized the streaming inside the viscous boundary layer near the wall [4]. Rayleigh's slip-velocity formalism was later generalized to curved surfaces moving in the normal direction $[5,6]$, to flat surfaces moving in arbitrary directions [7], and to curved surfaces with arbitrary velocity [8]. Eckart found in 1948 that acoustic streaming also can be induced by sound attenuation in the bulk [9]. This effect is mainly considered important for systems much larger than the acoustic wavelength $[10,11]$, but as shown recently, it can be significant on the scale of a single wavelength for rotating acoustics [12].

Acoustic streaming is a truly ubiquitous phenomenon observed not only in Newtonian fluids, but also in superfluid helium [13] and non-Newtonian viscoelastic liquids [14]. It is used in many different applications: thermoacoustic engines [15], enhancement of electrodedeposition [16], mixing in microfluidics [17,18], enhancement of particle trapping [19,20], micropumping [21], biofouling removal [22], and lysing of vesicles [23]. Given its widespread appearance, a fundamental question naturally arises: is it possible to suppress acoustic streaming? In one experimental study, streaming was suppressed by pulsed actuation [24], but the underlying mechanism remains unclear [25]. For steady actuation, experimental and theoretical studies have shown that steady streaming may be suppressed in inhomogeneous fluids inside a microchannel [26-28]. But can streaming in the conventional homogeneous steady case be suppressed?

In this Letter, using the experimentally validated numerical modeling of Refs. [27,29], we demonstrate that for confined homogeneous fluids, the steady acoustic streaming can be suppressed by more than two orders of magnitude in large parts of the bulk by optimizing the shape of the confinement. This discovery not only provides physical insight into a time-honored fundamental phenomenon in fluid dynamics, but it is also of considerable interest in the field of microscale acoustofluidics, where ultrasound fields routinely are used to handle suspended microparticles. Acoustically, a suspended spherical particle of radius $a$ is affected by two forces: the acoustic radiation force that scales with $a^{3}$ and tends to focus particles at the acoustic nodal planes; and the streaming-induced drag force that scales with $a$ and by virtue of the streaming vortices tends to defocus particles. Consequently, there exists a lower limit of $a$ that allows controlled handling by the radiation force; $a_{\min } \approx 1 \mu \mathrm{m}$ for dilute aqueous solutions of polystyrene particles at $2 \mathrm{MHz}[30,31]$. A suppression of the acoustic streaming would enable a desirable controlled handling of nanoparticles, such as bacteria, viruses, and exosomes.

Modeling the acoustofluidic fields.-To optimize the shape, efficient computation of the acoustofluidic fields is required. We use the method described in Refs. [8,32], where the viscous boundary layer is taken into account analytically without resolving it numerically. We consider a domain $\Omega$ with hard boundary walls, see Fig. 1, containing a homogeneous and quiescent fluid of dynamic viscosity $\eta_{\mathrm{fl}}$, bulk viscosity $\eta_{\mathrm{fl}}^{b}$, density $\rho_{\mathrm{fl}}$, and sound speed $c_{\mathrm{fl}}$ at pressure $p_{\mathrm{fl}}$. An acoustic field is created by letting the 

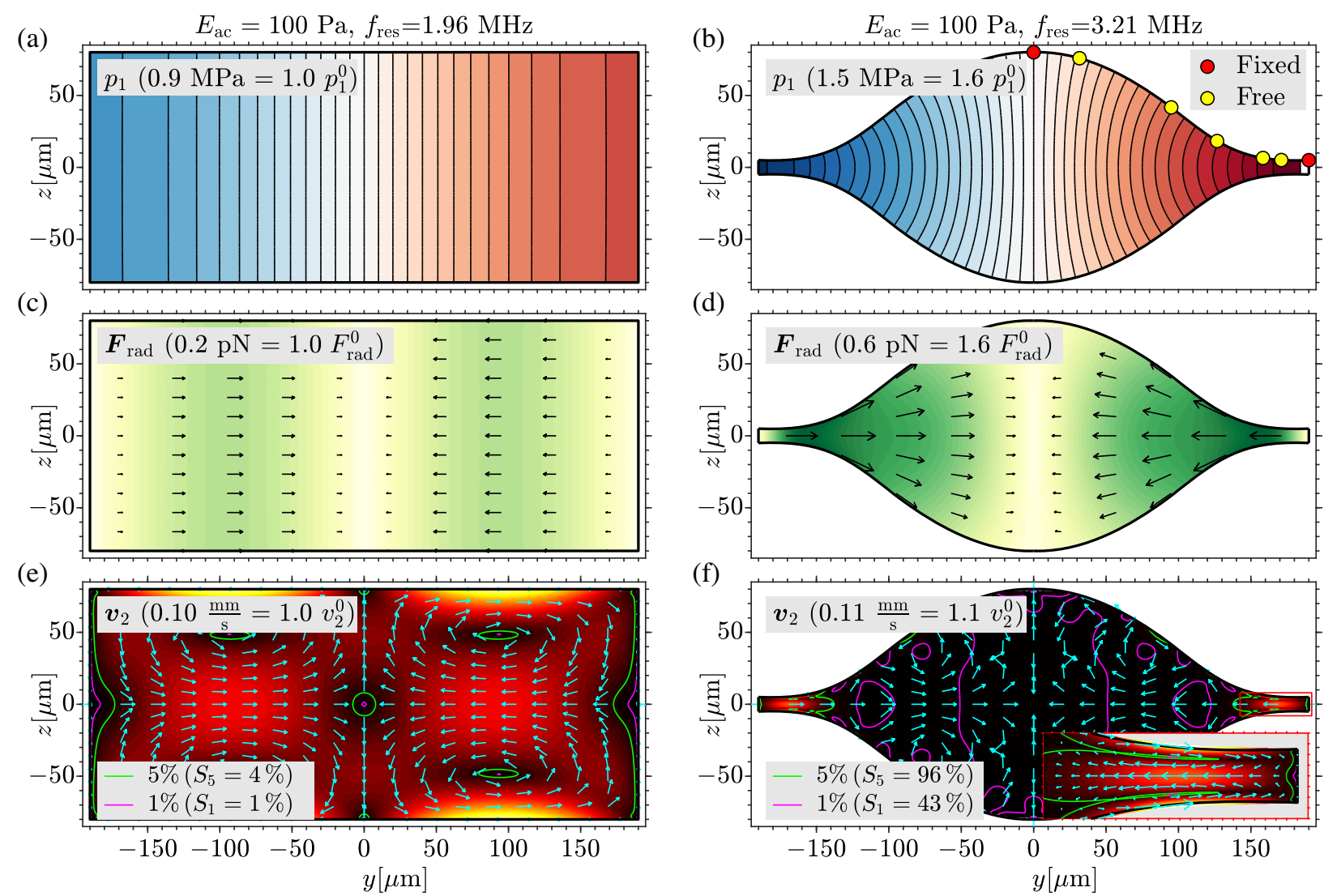

FIG. 1. Simulation results for $\mathrm{MHz}$ acoustics at $E_{\mathrm{ac}}=100 \mathrm{~Pa}$ in straight microchannels with a rectangular and a shape-optimized cross section. (a),(b) The acoustic pressure $p_{1}$ from $-1.5 \mathrm{MPa}$ (light blue) to $+1.5 \mathrm{MPa}$ (dark red). The shape in (b) is defined by a spline interpolation between the colored points, where the $z$ coordinate of the yellow points are free in the optimization, and the red points are fixed. (c),(d) The radiation force $\boldsymbol{F}_{\text {rad }}$ (black arrows) on 1- $\mu$ m-diameter polystyrene particles from 0 (white) to 0.6 pN (dark green). (e),(f) The acoustic streaming $\boldsymbol{v}_{2}$ (cyan unit arrows) from 0 (black) to 0.11 (mm/s) (light yellow). The two contours mark $1 \%$ (magenta) and $5 \%$ (green) of the characteristic streaming speed $v_{2}^{0}$ from Eq. (8).

boundary oscillate harmonically with the angular frequency $\omega$ around its equilibrium position $\partial \Omega$ with a prescribed displacement $\boldsymbol{u}^{\mathrm{bdr}}(\boldsymbol{r}, t)$ expressed as the real part of the complex-valued displacement amplitude $\boldsymbol{u}_{1}^{\mathrm{bdr}}(\boldsymbol{r})$,

$$
\boldsymbol{u}^{\mathrm{bdr}}(\boldsymbol{r}, t)=\operatorname{Re}\left[\boldsymbol{u}_{1}^{\mathrm{bdr}}(\boldsymbol{r}) e^{-i \omega t}\right], \quad \text { with } \quad i=\sqrt{-1} .
$$

The pressure $p$ is written as a perturbation series, and likewise for the density $\rho$ and the fluid velocity $\boldsymbol{v}$,

$$
p(\boldsymbol{r}, t)=p_{\mathrm{fl}}+\operatorname{Re}\left[p_{1}(\boldsymbol{r}) e^{-i \omega t}\right]+p_{2}(\boldsymbol{r}) .
$$

All first-order fields (subscript "1") oscillate harmonically with the angular frequency $\omega$, whereas all second-order fields (subscript " 2 ") are steady, being averaged in time over a full oscillation period $2 \pi / \omega$.

The first-order complex-valued acoustic pressure amplitude $p_{1}(\boldsymbol{r})$ satisfies the Helmholtz equation in the bulk $\Omega$ and a boundary-layer boundary condition at $\partial \Omega$ in terms of the inward normal derivative $\partial_{\perp}=-\boldsymbol{n} \cdot \boldsymbol{\nabla}$ and the outwardpointing normal vector $\boldsymbol{n}$ [8],

$$
\begin{gathered}
\nabla^{2} p_{1}+k_{c}^{2} p_{1}=0, \quad \text { inside } \Omega, \\
{\left[\partial_{\perp}+\frac{i}{k_{s}}\left(k_{c}^{2}+\partial_{\perp}^{2}\right)\right] p_{1}=\frac{-\rho_{\mathrm{fl}} \omega^{2}}{1-i \Gamma_{\mathrm{fl}}}\left(\boldsymbol{n}+\frac{i}{k_{s}} \boldsymbol{\nabla}\right) \cdot \boldsymbol{u}_{1}^{\mathrm{bdr}},}
\end{gathered}
$$$$
\text { at the boundary } \partial \Omega \text {. }
$$

Here, $k_{c}=\left(1+\frac{1}{2} i \Gamma_{\mathrm{fl}}\right) k_{0}$ is the complex-valued compressional wave number with real part $k_{0}=\left(\omega / c_{\mathrm{fl}}\right), \Gamma_{\mathrm{fl}}=$ $\frac{1}{2}\left[\frac{4}{3}+\left(\eta_{\mathrm{fl}}^{b} / \eta_{\mathrm{fl}}\right)\right]\left(k_{0} \delta\right)^{2} \ll 1$ is the acoustic damping coefficient, and $k_{s}=[(1+i) / \delta]$ is the shear wave number given by the viscous boundary-layer width $\delta=\sqrt{\left(2 \eta_{\mathrm{fl}} / \omega \rho_{\mathrm{fl}}\right)}$ with $k_{0} \delta \ll 1$. From $p_{1}$, we obtain the acoustic velocity $\boldsymbol{v}_{1}$ and density $\rho_{1}$ outside the boundary layer [8],

$$
\boldsymbol{v}_{1}=\frac{-i\left(1-i \Gamma_{\mathrm{fl}}\right)}{\omega \rho_{\mathrm{fl}}} \nabla p_{1}, \quad \rho_{1}=\rho_{\mathrm{fl}} \kappa_{\mathrm{fl}} p_{1},
$$

with the compressibility $\kappa_{\mathrm{fl}}=\left(1 / \rho_{\mathrm{fl}} c_{\mathrm{fl}}^{2}\right)$. The average acoustic energy density $E_{\text {ac }}$ in $\Omega$ of volume $\mathcal{V}_{\Omega}$ is, 


$$
E_{\mathrm{ac}}=\int_{\Omega}\left[\frac{1}{4} \kappa_{\mathrm{fl}}\left|p_{1}\right|^{2}+\frac{1}{4} \rho_{\mathrm{fl}}\left|\boldsymbol{v}_{1}\right|^{2}\right] \frac{d V}{\mathcal{V}_{\Omega}},
$$

where $\left|p_{1}\right|^{2}=p_{1} p_{1}^{*}$ and $\left|\boldsymbol{v}_{1}\right|^{2}=\boldsymbol{v}_{1} \cdot \boldsymbol{v}_{1}^{*}$ with " *" denoting the complex conjugate. The second-order steady acoustic streaming velocity $\boldsymbol{v}_{2}$ outside the viscous boundary layer is a Stokes flow with the slip velocity $v_{2}^{\text {slip }}$ at the boundary [8],

$$
\mathbf{0}=-\boldsymbol{\nabla} p_{2}+\eta_{\mathrm{fl}} \nabla^{2} \boldsymbol{v}_{2}+\frac{\Gamma_{\mathrm{fl}} \omega}{2 c_{\mathrm{fl}}^{2}} \operatorname{Re}\left(p_{1}^{*} \boldsymbol{v}_{1}\right),
$$

with $\quad 0=\boldsymbol{\nabla} \cdot \boldsymbol{v}_{2} \quad$ in $\Omega, \quad$ and $\quad \boldsymbol{v}_{2}=\boldsymbol{v}_{2}^{\text {slip }}$ at $\partial \Omega$.

The body force $\left(\Gamma_{\mathrm{fl}} \omega / 2 c_{\mathrm{fl}}^{2}\right) \operatorname{Re}\left(p_{1}^{*} \boldsymbol{v}_{1}\right)$ responsible for the bulk-driven streaming is included in the simulations, but turns out to be negligible. For the slip velocity $v_{2}^{\text {slip }}$, we use expression (55) of Ref. [8] (see the Supplemental Material [33]) for an oscillating, curved surface with a curvature radius much larger than the boundary-layer width $\delta$.

The time-averaged forces acting on a suspended particle of radius $a$ and velocity $\boldsymbol{v}_{\mathrm{pa}}$ are the Stokes drag force $\boldsymbol{F}_{\mathrm{drag}}$ and the acoustic radiation force $\boldsymbol{F}_{\text {rad }}$ [42],

$$
\begin{aligned}
& \boldsymbol{F}_{\mathrm{drag}}=6 \pi \eta_{\mathrm{fl}} a\left(\boldsymbol{v}_{2}-\boldsymbol{v}_{\mathrm{pa}}\right), \\
& \boldsymbol{F}_{\mathrm{rad}}=-\boldsymbol{\nabla}\left[\frac{4 \pi a^{3}}{3}\left(\frac{f_{0}}{4} \kappa_{\mathrm{fl}}\left|p_{1}\right|^{2}-\frac{3 f_{1}}{8} \rho_{\mathrm{fl}}\left|\boldsymbol{v}_{1}\right|^{2}\right)\right],
\end{aligned}
$$

where $f_{0}$ and $f_{1}$ are the monopole and dipole scattering coefficients for the particle. Parameters are listed in the Supplemental Material Tables II-V [33].

Shape optimization for suppression of acoustic streaming.-Experimental and theoretical studies show that acoustophoresis in long straight channels is well described as being translationally invariant, even if the setup exhibits only local translational invariance, say, above the transducer [43-45]. In the following, we therefore assume translational invariance along the $x$ axis, and consider microchannels with different $y-z$ cross sections, see Fig. 1. To quantify the comparison between these channels, we use the classical results for a standing half-wave resonance $p_{1}=p_{a} \sin \left(k_{0} y\right)$ in a rectangular cross section, for which $E_{\mathrm{ac}}=\frac{1}{4} \kappa_{\mathrm{fl}} p_{a}^{2}$, the slip velocity $\boldsymbol{v}_{2}^{\text {slip }}=$ $\left(3 E_{\mathrm{ac}} / 2 \rho_{\mathrm{fl}} c_{\mathrm{fl}}\right) \sin \left(2 k_{0} y\right) \boldsymbol{e}_{y}$ [3], and the acoustic radiation force $\boldsymbol{F}_{\text {rad }}=-4 \pi a^{3} k_{0} \Phi E_{\mathrm{ac}} \sin \left(2 k_{0} y\right) \boldsymbol{e}_{y}$, with the acoustic contrast factor $\Phi=\frac{1}{3} f_{0}+\frac{1}{2} f_{1}$ [46]. From this, we introduce three characteristic scaling quantities based on the acoustic energy density $E_{\mathrm{ac}}(5)$ : the acoustic pressure $p_{1}^{0}$, the streaming speed $v_{2}^{0}$, and the radiation force $F_{\text {rad }}^{0}$,

$$
p_{1}^{0}=\sqrt{\frac{4 E_{\mathrm{ac}}}{\kappa_{\mathrm{fl}}}}, \quad v_{2}^{0}=\frac{3 E_{\mathrm{ac}}}{2 \rho_{\mathrm{fl}} c_{\mathrm{fl}}}, \quad F_{\mathrm{rad}}^{0}=4 \pi a^{3} k_{0} \Phi E_{\mathrm{ac}} .
$$

To optimize the shape for suppression of acoustic streaming, we define a cost function $\mathcal{C}$ that penalizes large $\left|\boldsymbol{v}_{2}\right|$,

$$
\mathcal{C}=\frac{1}{v_{2}^{0}} \int_{\Omega}\left|\boldsymbol{v}_{2}\right| \frac{d V}{\mathcal{V}_{\Omega}} .
$$

The suppression of the streaming is quantified by the suppression parameter $S_{q}$, the volumetric fraction in which $\left|\boldsymbol{v}_{2}\right|$ is smaller than the percentage $q$ of $v_{2}^{0}$,

$S_{q}=\int_{\Omega} \Theta\left(\frac{q v_{2}^{0}}{100}-\left|\boldsymbol{v}_{2}\right|\right) \frac{d V}{\mathcal{V}_{\Omega}}, \quad \Theta(x)= \begin{cases}0, & \text { for } x<0, \\ 1, & \text { for } x>0 .\end{cases}$

For a given shape, we evaluate the cost function $\mathcal{C}$ by a numerical two-step simulation in COMSOL MULTIPHYSICS [47], see Refs. [8,30,32]: (1) we compute $p_{1}$ from Eq. (3) in the idealized case of a prescribed wall displacement $\boldsymbol{u}_{1}^{\mathrm{bdr}}=d_{0} \boldsymbol{e}_{y}$, where $d_{0}$ is chosen such that $E_{\mathrm{ac}}=100 \mathrm{~Pa}$. (2) We solve Eq. (6) for $\boldsymbol{v}_{2}$ with $\boldsymbol{v}_{2}^{\text {slip }}$ calculated from $p_{1}$.

The shape is constrained to have width $W_{0}=380 \mu \mathrm{m}$ and height $H_{0}=160 \mu \mathrm{m}$, and to be symmetric in $y$ and $z$, see Fig. 1(b). The upper right edge is represented by a cubic spline interpolation through seven points $\left(y_{j}, z_{j}\right)$, $j=0,1, \ldots, 6$, where the $y$ positions $y_{j}$ are fixed at $\left[0, \frac{1}{6}, \frac{3}{6}, \frac{4}{6}, \frac{5}{6}, \frac{9}{10}, 1\right]\left(W_{0} / 2\right)$, and where the $z$ positions of the end points are fixed at $z_{0}=\frac{1}{2} H_{0}$ and $z_{6}=\frac{1}{2} h_{0}, h_{0}=$ $10 \mu \mathrm{m}$ being the height of the channel at the neck $y_{6}=\frac{1}{2} W_{0}$. The optimization algorithm minimizes the cost function $\mathcal{C}$ (9) by varying the five free heights $z_{1}-z_{5}$ with the constraint $\frac{1}{2} h_{0} \leq z_{j} \leq \frac{1}{2} H_{0}$. This optimization is implemented in MATLAB [48] using the routine FMINSEARCHBND [49] that calls COMSOL. It typically requires $~ 200$ iterations, each taking 5 seconds on a workstation with a 3.5-GHz Intel Xeon CPU E5-1650 v2 dual-core processor and with a memory of 128 GB RAM.

In Fig. 1, simulation results are shown for the wellstudied rectangular shape [30] and compared to the results for the optimized spline shape. For the latter, the acoustic streaming is dramatically suppressed, whereas the radiation force remains large. Quantitatively, we obtain from Eq. (10) the streaming-suppression parameters $S_{5}=96 \%$ and $S_{1}=$ $38 \%$ for the optimized shape, and $S_{5}=4 \%$ and $S_{1}=0.6 \%$ for the rectangle.

In Fig. 2(a), we show the family of optimized shapes obtained as above, but for the different maximum heights $z_{0}=[0.1,0.2, \ldots, 1.5] H_{0} / 2$. In Fig. 2(b), we plot $p_{1}$ along the upper boundary and note that it is nearly linear along a large part of the arc length for all the optimized shapes. This may be explained by inspecting the simplified expression for the slip velocity $v_{2}^{\text {slip }}$ adapted from Eq. (61) in Ref. [8] to the 2D standing-wave resonance considered here, see the Supplemental Material [33], 


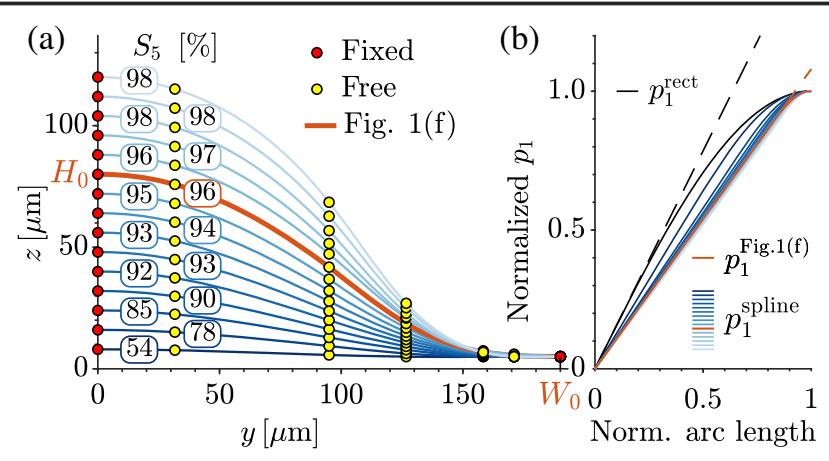

FIG. 2. (a) The optimized shapes, each defined by two fixed (red) and five free (yellow) points, obtained as in Fig. 1 but for different maximum height constraints ranging from $0.1 H_{0}$ (dark blue) to $1.5 H_{0}$ (light blue). The curves are labeled by the suppression parameter $S_{5}$, Eq. (10). The thick orange shape is the one shown in Fig. 1(f). (b) The acoustic pressure $p_{1}$ versus arc length along the boundaries shown in (a) using the same color scheme. The black curve shows the pressure obtained in the rectangular cross section, where $p_{1}^{\text {rect }} \propto \sin \left(\pi y / W_{0}\right)$. The dashed lines are selected tangents.

$$
\boldsymbol{v}_{2 \|}^{\text {slip }} \approx-\frac{3}{8 \omega} \nabla_{\|}\left|\boldsymbol{v}_{1 \|}\right|^{2}, \quad v_{2 \perp}^{\text {slip }} \approx 0
$$

We see that $v_{2 \|}^{\text {slip }}$ is small if $\left|v_{1 \|}\right|$ is approximately constant along the boundary, and since $\boldsymbol{v}_{1 \|} \propto \nabla_{\|} p_{1}$, this is equivalent with $p_{1}$ varying linearly along the boundary. Remarkably, as seen in Fig. 2(b), this linearity is maintained along nearly $90 \%$ of the optimized boundaries, but eventually, due to the no-slip boundary condition, the pressure gradient must tend to zero at the end point $\left(\frac{1}{2} W_{0}, \frac{1}{2} h_{0}\right)$. The last $10 \%$ of the boundary therefore generates streaming, so by forming narrow necks there, the streaming becomes localized in a small region.

In Fig. 3(a), we study the importance of the narrow necks of the shape in Fig. 1(f) by cutting them off, leaving $90 \%$ of the width, $-(9 / 20)<\left(y / W_{0}\right)<(9 / 20)$. In this case, the streaming is still suppressed: with $S_{5}=63 \%$ and $S_{1}=3 \%$, it is worse compared to Fig. 1(f) with the necks, where $S_{5}=96 \%$ and $S_{1}=38 \%$, but much better than for the rectangle of Fig. 1(e), where $S_{5}=4 \%$ and $S_{1}=1 \%$. As it might prove difficult to fabricate the exact optimized shape, we study in Fig. 3(b) a generic shape with a narrow neck and a wide bulk given by a cosine, $z(y)= \pm(h / 2) \pm$ $\left[\left(H_{0} / 2\right)-(h / 2)\right]\left[\frac{1}{2}+\frac{1}{2} \cos \left(2 \pi y / W_{0}\right)\right]$. Here, the neck height $h$ is the only free parameter. Using the cost function $\mathcal{C}$ again, the optimal value is found to be $h=3.14 \mu \mathrm{m}$ with a fair streaming suppression of $S_{5}=55 \%$ and $S_{1}=3 \%$. See Table I of the Supplemental Material [33] for more details.

Particle focusing.- In the conventional rectangular shape, the minimum radius $a_{\min }$ of particles that can be focused is estimated by equating $F_{\text {rad }}^{0}$ and the drag force
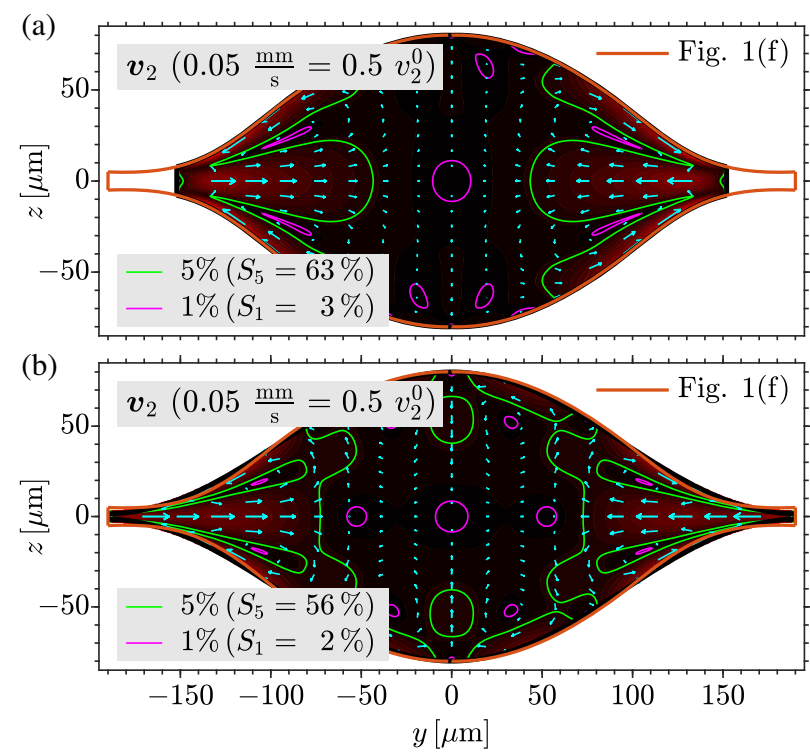

FIG. 3. The acoustic streaming velocity $\boldsymbol{v}_{2}$ (cyan arrows), its magnitude $v_{2}$ from 0 (black) to $0.05(\mathrm{~mm} / \mathrm{s})$ (light yellow), and the $5 \%$ (green) and $1 \%$ (magenta) contour lines of $v_{2}$ as in Fig. 1(f), but for two different hard-wall shapes. (a) The optimized shape Fig. 1(f) (orange dashed curve) with its necks cut off, and (b) the optimized cosine shape.

$6 \pi \eta_{\mathrm{fl}} a v_{2}^{0} \quad$ [30,31], Eq. (8): $\quad a_{\mathrm{min}}^{\mathrm{rect}}=\sqrt{\left(9 \eta_{\mathrm{fl}} / 4 \rho_{\mathrm{fl}} \omega \Phi\right)}=$ $0.9 \mu \mathrm{m}$ for polystyrene particles with $\Phi=0.16$ in water at $f=1.95 \mathrm{MHz}$. In the optimized shape, the streaming is suppressed by $95 \%$ at $f=3.02 \mathrm{MHz}$, leading to a substantial sixfold reduction of $a_{\text {min }}$ to $a_{\text {min }}^{\text {opt }} \approx 0.15 \mu \mathrm{m}$. See the Supplemental Material [33] for acoustophoresis simulations of 300-, 1000-, and 3000-nm-diameter particles in shapeoptimized and rectangular channels. As expected, 300-nm particles focus only in the shape-optimized channel.

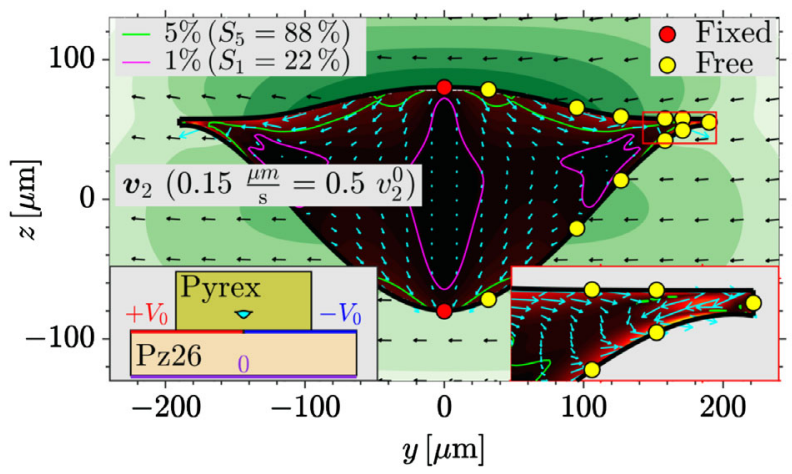

FIG. 4. The streaming field (black-to-yellow contour plot) in the fluid channel and the displacement field (light-to-dark-green contour plot) in the surrounding glass $(3 \times 1.3 \mathrm{~mm})$ in a fulldevice simulation including a Pz26 transducer $(5 \times 1 \mathrm{~mm})$ with split top $\left( \pm V_{0}\right)$ and grounded bottom (0) electrode (bottom-left inset). The neck is shown in the lower-right inset. The optimized fluid channel shape is obtained by varying the $z$ coordinates of the 11 yellow points (the 2 red being fixed), see the Supplemental Material for details [33]. 
Solids and transducers. - For a more realistic model, we embed the microchannel in a rectangular glass block mounted on a piezoelectric transducer with a split top electrode for antisymmetric ac voltage actuation [50], see Fig. 4 (lower left inset). As the up-down symmetry is broken, we optimize the shape with 11 free and 2 fixed points, still using the cost function $\mathcal{C}$. The resulting streaming is significantly suppressed, $S_{5}=88 \%$ and $S_{1}=22 \%$, see the Supplemental Material [33] for details.

Conclusion.-By exploiting effective boundary conditions [8], we have implemented an optimization algorithm that computes the shape of an acoustic cavity, which at resonance has the remarkable property that the acoustic streaming is dramatically suppressed relative to the conventional rectangular cavity. Notably, the acoustic pressure amplitude and the acoustic radiation force acting on suspended particles are not suppressed, and therefore, the optimized cavity shape is particularly ideal for applications involving controlled acoustophoretic handling of $\mathrm{nm}$-sized particles. This application aspect will be studied in future work. Note that our method is not limited to hard materials such as glass. Even soft polymers may be used, relying on the whole-system resonance principle [51], perhaps in combination with the recently developed method of structured microfluidic fibers [52].

By shape optimization, we have gained insight in fundamental acoustofluidics, and shown how the ubiquitous acoustic streaming is suppressed by ensuring a linear acoustic pressure profile along the wall. We have used this insight to suggest practical applications. By applying other optimization methods, say topology optimization [53], or other cost functions, such as one based on acoustophoretic force fields, our method may be extended to other fundamental studies within nonlinear acoustics.

*jasoba@fysik.dtu.dk

bruus@fysik.dtu.dk

[1] M. Faraday, On a peculiar class of acoustical figures; and on certain forms assumed by groups of particles upon vibrating elastic surfaces, Phil. Trans. R. Soc. London 121, 299 (1831).

[2] V. Dvořák, Ueber die akustische Anziehung und Abstossung, Ann. Phys. Chem. 157, 42 (1876).

[3] Lord Rayleigh, On the circulation of air observed in Kundt's tubes, and on some allied acoustical problems, Phil. Trans. R. Soc. London 175, 1 (1884).

[4] H. Schlichting, Berechnung ebener periodischer grenzeschichtströmungen, Phys. Z. 33, 327 (1932).

[5] W. L. Nyborg, Acoustic streaming near a boundary, J. Acoust. Soc. Am. 30, 329 (1958).

[6] T. G. Wang, E. H. Trinh, A. P. Croonquist, and D. D. Elleman, Shapes of Rotating Free Drops: Spacelab Experimental Results, Phys. Rev. Lett. 56, 452 (1986).

[7] J. Vanneste and O. Bühler, Streaming by leaky surface acoustic waves, Proc. R. Soc. A 467, 1779 (2011).
[8] J. S. Bach and H. Bruus, Theory of pressure acoustics with viscous boundary layers and streaming in curved elastic cavities, J. Acoust. Soc. Am. 144, 766 (2018).

[9] C. Eckart, Vortices and streams caused by sound waves, Phys. Rev. 73, 68 (1948).

[10] P. J. Westervelt, The theory of steady rotational flow generated by a sound field, J. Acoust. Soc. Am. 25, 60 (1953).

[11] A. Riaud, M. Baudoin, O. Bou Matar, L. Becerra, and J.-L. Thomas, Selective Manipulation of Microscopic Particles with Precursor Swirling Rayleigh Waves, Phys. Rev. Applied 7, 024007 (2017).

[12] J. S. Bach and H. Bruus, Bulk-driven acoustic streaming at resonance in closed microcavities, Phys. Rev. E 100, 023104 (2019).

[13] J. Rooney, C. Smith, and R. Carey, Acoustic streaming in superfluid-helium, J. Acoust. Soc. Am. 72, 245 (1982).

[14] G. Vishwanathan and G. Juarez, Steady streaming flows in viscoelastic liquids, J. Non-Newton. Fluids 271, 104143 (2019).

[15] I. A. Ramadan, H. Bailliet, and J.-C. Valiere, Experimental investigation of the influence of natural convection and endeffects on Rayleigh streaming in a thermoacoustic engine, J. Acoust. Sos. Am. 143, 361 (2018).

[16] J. G. Kaufmann, M. P. Y. Desmulliez, Y. Tian, D. Price, M. Hughes, N. Strusevich, C. Bailey, C. Liu, and D. Hutt, Megasonic agitation for enhanced electrodeposition of copper, Microsyst. Technol. 15, 1245 (2009).

[17] K. Sritharan, C. Strobl, M. Schneider, A. Wixforth, and Z. Guttenberg, Acoustic mixing at low Reynold's numbers, Appl. Phys. Lett. 88, 054102 (2006).

[18] P.-H. Huang, Y. Xie, D. Ahmed, J. Rufo, N. Nama, Y. Chen, C. Y. Chan, and T. J. Huang, An acoustofluidic micromixer based on oscillating sidewall sharp-edges, Lab Chip 13, 3847 (2013).

[19] B. Hammarström, T. Laurell, and J. Nilsson, Seed particle enabled acoustic trapping of bacteria and nanoparticles in continuous flow systems, Lab Chip 12, 4296 (2012).

[20] I. Leibacher, P. Hahn, and J. Dual, Acoustophoretic cell and particle trapping on microfluidic sharp edges, Microfluid. Nanofluid. 19, 923 (2015).

[21] P.-H. Huang, N. Nama, Z. Mao, P. Li, J. Rufo, Y. Chen, Y. Xie, C.-H. Wei, L. Wang, and T. J. Huang, A reliable and programmable acoustofluidic pump powered by oscillating sharp-edge structures, Lab Chip 14, 4319 (2014).

[22] S. K. R. S. Sankaranarayanan, S. Cular, V. R. Bhethanabotla, and B. Joseph, Flow induced by acoustic streaming on surface-acoustic-wave devices and its application in biofouling removal: A computational study and comparisons to experiment, Phys. Rev. E 77, 066308 (2008).

[23] P. Marmottant and S. Hilgenfeldt, Controlled vesicle deformation and lysis by single oscillating bubbles, Nature (London) 423, 153 (2003).

[24] M. Hoyos and A. Castro, Controlling the acoustic streaming by pulsed ultrasounds, Ultrasonics 53, 70 (2013).

[25] P. B. Muller and H. Bruus, Theoretical study of timedependent, ultrasound-induced acoustic streaming in microchannels, Phys. Rev. E 92, 063018 (2015).

[26] P. Augustsson, J. T. Karlsen, H.-W. Su, H. Bruus, and J. Voldman, Iso-acoustic focusing of cells for size-insensitive 
acousto-mechanical phenotyping, Nat. Commun. 7, 11556 (2016).

[27] J. T. Karlsen, W. Qiu, P. Augustsson, and H. Bruus, Acoustic Streaming and Its Suppression in Inhomogeneous Fluids, Phys. Rev. Lett. 120, 054501 (2018).

[28] W. Qiu, J. T. Karlsen, H. Bruus, and P. Augustsson, Experimental Characterization of Acoustic Streaming in Gradients of Density and Compressibility, Phys. Rev. Applied 11, 024018 (2019).

[29] J. T. Karlsen, P. Augustsson, and H. Bruus, Acoustic Force Density Acting on Inhomogeneous Fluids in Acoustic Fields, Phys. Rev. Lett. 117, 114504 (2016).

[30] P. B. Muller, R. Barnkob, M. J. H. Jensen, and H. Bruus, A numerical study of microparticle acoustophoresis driven by acoustic radiation forces and streaming-induced drag forces, Lab Chip 12, 4617 (2012).

[31] R. Barnkob, P. Augustsson, T. Laurell, and H. Bruus, Acoustic radiation- and streaming-induced microparticle velocities determined by microparticle image velocimetry in an ultrasound symmetry plane, Phys. Rev. E 86, 056307 (2012).

[32] N. R. Skov, J. S. Bach, B. G. Winckelmann, and H. Bruus, 3D modeling of acoustofluidics in a liquid-filled cavity including streaming, viscous boundary layers, surrounding solids, and a piezoelectric transducer, AIMS Math. 4, 99 (2019).

[33] See the Supplemental Material at http://link.aps.org/ supplemental/10.1103/PhysRevLett.124.214501 for derivations of Eqs. (6b) and (11), lists of material parameters, a description of the shape optimization for a fluid domain embedded in an elastic solid, and links to simulation animations of acoustophoretic nanoparticle focusing in the shape-optimized and rectangular channel, which includes Refs. [34-41].

[34] Ferroperm Matdat 2017, Meggit A/S, Porthusvej 4, DK3490 Kvistgaard, Denmark, https://www.meggittferroperm .com/materials/ (2020).

[35] P. Hahn and J. Dual, A numerically efficient damping model for acoustic resonances in microfluidic cavities, Phys. Fluids 27, 062005 (2015).

[36] Glass Silicon Constraint Substrates, CORNING, Houghton Park C-8, Corning, NY 14831, USA, http://www .valleydesign.com/Datasheets/Corning\%20Pyrex\%207740 .pdf (2016).

[37] P. B. Muller and H. Bruus, Numerical study of thermoviscous effects in ultrasound-induced acoustic streaming in microchannels, Phys. Rev. E 90, 043016 (2014).
[38] CRC Handbook of Chemistry and Physics, edited by W. M. Haynes (CRC Press, Boca Raton, FL, 2014), 95th ed..

[39] Seven Simulation Videos (68 MB) by J. S. Bach and H. Bruus (2020), http://bruus-lab.dk/TMF/publications/ pub2019/Bach_seven_animation_videos.zip.

[40] M. R. Maxey and J. J. Riley, Equation of motion for a small rigid sphere in a nonuniform flow, Phys. Fluids 26, 883 (1983).

[41] J. S. Hansen, J. C. Dyre, P. Daivis, B. D. Todd, and H. Bruus, Continuum nanofluidics, Langmuir 31, 13275 (2015).

[42] M. Settnes and H. Bruus, Forces acting on a small particle in an acoustical field in a viscous fluid, Phys. Rev. E 85, 016327 (2012).

[43] P. Augustsson, R. Barnkob, S. T. Wereley, H. Bruus, and T. Laurell, Automated and temperature-controlled micro-PIV measurements enabling long-term-stable microchannel acoustophoresis characterization, Lab Chip 11, 4152 (2011).

[44] P. B. Muller, M. Rossi, A. G. Marin, R. Barnkob, P. Augustsson, T. Laurell, C. J. Kähler, and H. Bruus, Ultrasound-induced acoustophoretic motion of microparticles in three dimensions, Phys. Rev. E 88, 023006 (2013).

[45] M. W. H. Ley and H. Bruus, Three-Dimensional Numerical Modeling of Acoustic Trapping in Glass Capillaries, Phys. Rev. Applied 8, 024020 (2017).

[46] K. Yosioka and Y. Kawasima, Acoustic radiation pressure on a compressible sphere, Acustica 5, 167 (1955).

[47] COMSOL Multiphysics 5.4, http://www.comsol.com (2018).

[48] Matlab 2019b, http://www.mathworks.com (2019).

[49] J. D'Errico, Matlab file exchange (2012), https:// www.mathworks.com/matlabcentral/fileexchange/8277fminsearchbnd-fminsearchcon (2020).

[50] W. N. Bodé, L. Jiang, T. Laurell, and H. Bruus, Microparticle acoustophoresis in aluminum-based acoustofluidic devices with PDMS covers, Micromachines 11, 292 (2020).

[51] R. P. Moiseyenko and H. Bruus, Whole-System Ultrasound Resonances as the Basis for Acoustophoresis in AllPolymer Microfluidic Devices, Phys. Rev. Applied 11, 014014 (2019).

[52] R. Yuan, J. Lee, H.-W. Su, E. Levy, T. Khudiyev, J. Voldman, and Y. Fink, Microfluidics in structured multimaterial fibers, Proc. Natl. Acad. Sci. U.S.A. 115, E10830 (2018).

[53] L. Olesen, F. Okkels, and H. Bruus, A high-level programminglanguage implementation of topology optimization applied to steady-state Navier-stokes flow, Int. J. Numer. Methods Eng. 65, 975 (2006). 